\title{
Feza H. Remzi, MD leads Colorectal Surgery at Cleveland Clinic, Digestive Disease Institute, Cleveland, Ohio
}

\author{
James I. Merlino
}

(C) Springer-Verlag 2009

For 33 years, Victor W. Fazio, MD led the Department of Colorectal Surgery at Cleveland Clinic. But it was with great pleasure that he turned over the reins as Chairman of the Department late last year to Feza H. Remzi, MD, who he mentored for so many years (Fig. 1). Today, Dr. Remzi is an internationally renowned colorectal surgeon and a leader in the surgical treatment of inflammatory bowel disease and other colorectal conditions. He will lead a team that performs the highest volumes of J-pouch surgeries in the world for ulcerative colitis, and collaborates very closely with our gastroenterologists to manage these patients post-operatively. Dr. Fazio will remain in his position as Chairman of the Digestive Disease Institute.

The Cleveland Clinic Digestive Disease Institute is ranked one of the top Digestive Disease Centers in the United States (Fig. 2). The institute is one of the largest in the country and is also one of the first to unite all specialists in gastroenterology, hepatology, colorectal surgery, hepato-pancreato-biliary and transplant surgery, and nutrition within one unique, fully integrated model of care-aimed at optimizing the patient experience. The Cleveland Clinic Digestive Disease Institute is committed to a multidisciplinary approach to colorectal cancer care, providing excellent care through patient screening, education, detection, and treatment. Their team approach brings together the expertise of specialists in a wide range of disciplines including colorectal surgery, gastroenterology, medical oncology, radiation oncology, and genetic counseling. Together, they work with patients and their families

J. I. Merlino $(\square)$

Digestive Disease Institute, Cleveland Clinic Foundation, 9500 Euclid Avenue A-309, Cleveland, OH, USA

e-mail: merlinj@ccf.org

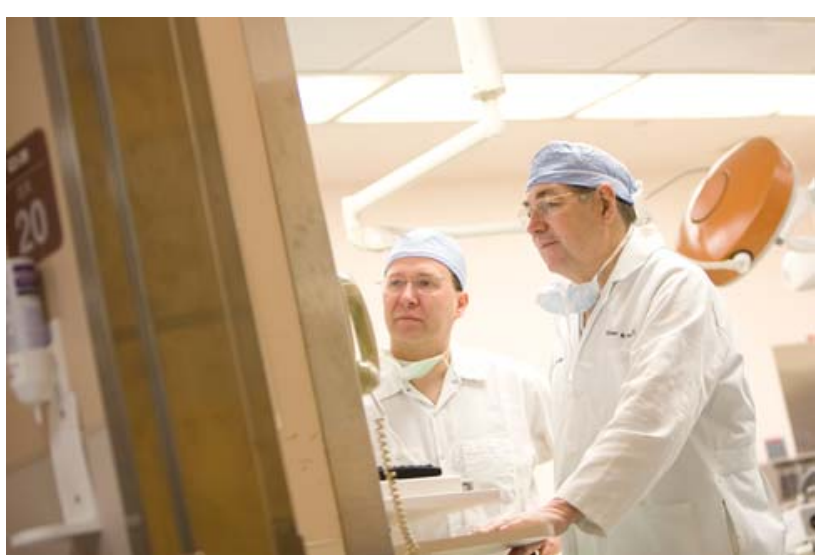

Fig. 1 Digestive Disease Institute Chair, Dr. Victor Fazio discusses a case with the new Chair of Colorectal Surgery, Dr. Feza Remzi

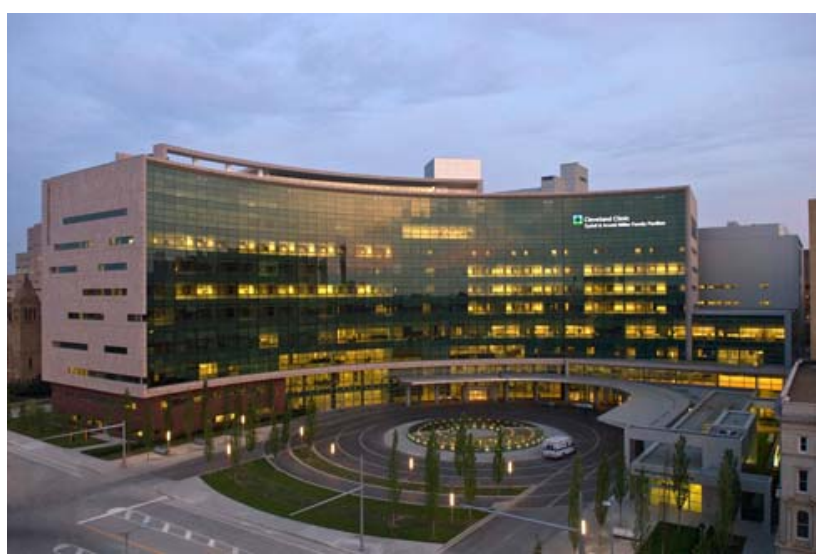

Fig. 2 The new Miller Pavilion at the Cleveland Clinic Foundation

to develop treatment plans tailored to their individual needs. This approach offers patients the greatest chance of cure and the best-possible quality of life. 
In 2008, the Digestive Disease Institute saw 1,635 Crohn's Disease patients, 893 Ulcerative Colitis patients, performed 147 liver transplants, and to date have performed 3,200 J-pouch operations. Currently, the DDI employees are 50 Gastroenterologists and Hepatologists, 19 Colorectal Surgeons, 13 Hepato-Pancreato-Biliary and Transplant Surgeons, 67 registered dietitians and dietetic technicians and nearly 500 nursing team members.

The Digestive Disease Institute, due to its participation in clinical trials and the excellent outcome of its surgical cases, receives worldwide referrals particularly for complex and severely affected patients. DDI is the largest national referral center for repairing failed pelvic pouches. Patients come from around the world to the Digestive Disease Institute for re-operations. Moving forward under Dr. Remzi's leadership, the DDI will continue to lead the world in laparoscopic surgery by pushing the envelope using smaller incisions, fewer ports, and different extraction sites. Advancing the use of robotics in colorectal surgery will also improve the overall patient experience. Recently, Dr. Remzi performed the world's first single-port colectomy in December 2007. Under Dr. Remzi's leadership, Dr. Daniel Geisler performed the World's first single-port proctocolectomy with J-pouch reconstruction in June 2009. To learn more about Cleveland Clinic's Digestive Disease Institute, visit http://www.clevelandclinic.org/digestive. 